\title{
Effect of Intraoperative Blood Loss on Perioperative Complications and Neurological Outcome in Adult Patients Undergoing Elective Brain Tumor Surgery
}

\author{
Vanitha Rajagopalan ${ }^{1}$ Rajendra Singh Chouhan ${ }^{1}$ Mihir Prakash Pandia ${ }^{1}$ Ritesh Lamsal ${ }^{1}$ \\ Girija Prasad Rath ${ }^{1}$ \\ ${ }^{1}$ Department of Neuroanaesthesiology and Critical Care, All India
Institute of Medical Sciences, New Delhi, India

\begin{abstract}
Address for correspondence Girija Prasad Rath, MD, DM, Department of Neuroanaesthesiology and Critical Care, Neurosciences Centre, All India Institute of Medical Sciences, New Delhi 110029, India (e-mail: girijarath@yahoo.co.in).
\end{abstract}

\begin{abstract}
Keywords

- blood loss

- brain tumor

- neurological outcome

- neurosurgery

- perioperative complications

Background Major blood loss during neurosurgery can lead to several complications, including life-threatening hemodynamic instabilities. Studies addressing these complications in patients undergoing intracranial tumor surgery are limited.

Materials and Methods During the study period, 456 patients who underwent elective craniotomy for brain tumor excision were categorized into four groups on the basis of estimated intraoperative blood volume loss: Group A (<20\%), Group B (20-50\%), Group C (>50-100\%), and Group D (more than estimated blood volume). The occurrence of various perioperative complications was correlated with these groups to identify if there was any association with the amount of intraoperative blood loss.

Results The average blood volume loss was $11 \% \pm 5.3 \%$ in Group A, $29.8 \% \pm 7.9 \%$ in Group B, 68.3\% $\pm 13.5 \%$ in Group C, and $129.1 \% \pm 23.9 \%$ in Group D. Variables identified as risk factors for intraoperative bleeding were female gender $(p<0.001)$, hypertension ( $p=0.008)$, tumor size $>5 \mathrm{~cm}(p<0.001)$, high-grade glioma $(p=0.004)$, meningioma $(p<0.001)$, mass effect $(p=0.002)$, midline shift $(p=0.014)$, highly vascular tumors documented on preoperative imaging $(p<0.001)$, extended craniotomy approach $(p=0.002)$, intraoperative colloids use $>1,000 \mathrm{~mL}(p<0.001)$, intraoperative brain bulge $(p=0.03)$, intraoperative appearance as highly vascular tumor $(p<0.001)$, and duration of surgery $>300$ minutes $(p<0.001)$.

Conclusions Knowledge of these predictors may help anesthesiologists anticipate major blood loss during brain tumor surgery and be prepared to mitigate these complications to improve patient outcome.
\end{abstract}

\section{Introduction}

Blood loss during neurosurgery varies in magnitude from minimal to massive. Excessive blood loss is strongly associated with in-hospital morbidity and mortality. ${ }^{1}$ Complications of transfusion translate into higher rates of sepsis, longer duration of hospital and intensive care unit (ICU) stay, and overall increased patient mortality. ${ }^{2-7}$ Several studies have identified complications associated with bleeding in patients with trauma, obstetric hemorrhage, cardiac surgery, liver transplantation, and oncological surgery. ${ }^{8-14}$ However, studies addressing these complications during intracranial tumor surgery are not available. Hence, we planned this study to correlate the amount of intraoperative bleeding with perioperative complications and neurological outcome in patients undergoing brain tumor surgery.
DOI https://doi.org/ $10.1055 / \mathrm{s}-0039-3399487$ ISSN 0976-3147.
License terms

$($ () (1) $\Theta \circledast$ 


\section{Materials and Methods}

After obtaining approval from the Institutional Ethics Committee and permission to access the patients' records, data of all adult patients between 18 and 60 years, who underwent elective surgery for brain tumor removal over a period of 1 year, were collected by reviewing the patients' medical records and intraoperative anesthesia charts.

Preoperative patient data, including age, sex, body weight, associated systemic illness, American Society of Anesthesiologists (ASA) physical status, type and site of lesion, the number of previous surgeries for the pathology, preoperative cardiorespiratory status, and preoperative baseline investigations, were collected. Estimated blood volume (EBV) of the individual patient was calculated on the basis of his or her gender (men: $70 \mathrm{~mL} / \mathrm{kg}$; women: $65 \mathrm{~mL} / \mathrm{kg}$ ).

Intraoperative anesthesia record and surgical notes were reviewed to collect data regarding type of anesthesia technique, surgical position, surgical procedure, hemodynamic stability, amount of blood loss, amount and type of intravenous (IV) fluid administered, amount of blood and blood components transfused, the use of vasopressors, the use of cell saver, and duration of surgery and anesthesia. Intraoperative complications such as hemodynamic instability, hypothermia, derangements in blood gases and acid-base status, dyselectrolytemia, arrhythmias, coagulation abnormality if any, and details of their management were noted. After completion of the surgery, it was also recorded whether the patient was extubated immediately or was mechanically ventilated.

Patients medical records were reviewed to note the development of postoperative complications within the first 48 hours related to blood loss and transfusion, such as anemia (hemoglobin $<8 \mathrm{~g} \%$ ), leukocytosis (total leukocyte count $>11,000 / \mathrm{mm}^{3}$ ), thrombocytopenia (platelet count $<100,000 /$ $\mathrm{mm}^{3}$ ), fever, coagulation abnormality, deranged hepatic/renal parameters, and further blood and component transfusion. Other complications such as neurological deficits, cardiovascular complications, pulmonary complications, infections (pulmonary, bloodstream, wound, meningitis, and sepsis), renal failure, electrolyte imbalance, and hemodynamic instability requiring inotrope/vasopressor were also recorded. Hematoma in the postoperative computed tomographic scan, need for reexploration for hematoma evacuation, need for tracheostomy, the number of days of mechanical ventilation, and days of ICU and hospital stay were also noted. Prolonged mechanical ventilation was defined as duration of mechanical ventilation $>48$ hours. Similarly, prolonged ICU stay was taken as ICU stay $>2$ days and prolonged hospital stay was considered as hospital stay $>7$ days. Patient condition at the time of discharge was noted to assign Glasgow Outcome Scale (GOS) grade to assess neurological outcome. GOS grade IV and V were considered "good outcome," whereas GOS Grade I, II, and III were included in "poor outcome."

All the patients were then categorized into four groups on the basis of their estimated intraoperative blood loss as follows:

- Group A: <20\% blood volume loss

- Group B: 20 to 50\% blood volume loss
- Group C: $>50$ to $100 \%$ blood volume loss

- Group D: blood loss exceeding estimated total blood volume

Occurrence of various perioperative complications was then correlated with the patient groups to identify if there was any association with the amount of intraoperative blood loss.

The primary objective of this study was to study the effects of intraoperative blood loss on perioperative complications and neurological outcome in adult patients undergoing elective brain tumor surgery. The secondary objectives were to identify various risk factors predicting intraoperative blood loss in these patients.

\section{Statistical Analysis}

The statistical analysis was performed using STATA 12.1 (College Stations, Texas, United States). Data are presented as number (\%)/mean \pm standard deviation/median (range) as appropriate. For univariate analysis, we used the chi-squared test and the Fisher's exact test (when one or more expected frequencies were $<5$ ) for categorical variables and the Student's $t$-test for continuous variables. Univariate analysis followed by multiple logistic regression analysis was done to identify risk factors and independent predictors for the occurrence of blood loss $>20 \%$. The results are reported as odds ratio (OR) and 95\% confidence interval (CI). p-Value $<0.05$ was considered statistically significant.

\section{Results}

During the study period, 456 patients who underwent elective craniotomy for brain tumor excision qualified for analysis as per the inclusion criteria, and their data were analyzed. After categorization into four groups based on the intraoperative estimated blood volume loss, there were 250 (54.8\%) patients in Group A, 154 (33.7\%) patients in Group B, $41(8.9 \%)$ patients in Group C, and 11 (2.4\%) patients in Group D.

Mean age of our study patients was $37.4 \pm 11.5$ years. Most of the patients were in the third and fourth decades of life (-Fig. 1). There was almost equal distribution of men and women (246 men [53.9\%] vs. 210 women [46.1\%]) (-Table 1). The majority of the patients belonged to ASA physical status I (76\%) (-Table 1). Hypertension (10.9\%) was the most common comorbidity followed by diabetes mellitus and hypothyroidism; multiple comorbidities were seen in 14 patients.

Most patients underwent surgery for excision of primary tumors (98.5\%). The tumors were commonly supratentorial (46.9\%) in location ( - Table 2). Histopathologically, about onethird of the patients had glioma (31.5\%), followed by meningioma (27.6\%) and schwannoma (16\%). Eighty-four patients (18.4\%) underwent redo surgeries. About 30\% patients had large ( $>5 \mathrm{~cm}$ size) and highly vascular tumors. A little more than three-fourths of our patients had mass effect preoperatively, while only $11.8 \%$ and $19.3 \%$ patients, respectively, had midline shift $>5 \mathrm{~mm}$ and hydrocephalus before surgery.

Nearly, all patients (98.5\%) underwent craniotomy under general anesthesia. There were only a few (1.5\%) patients who 


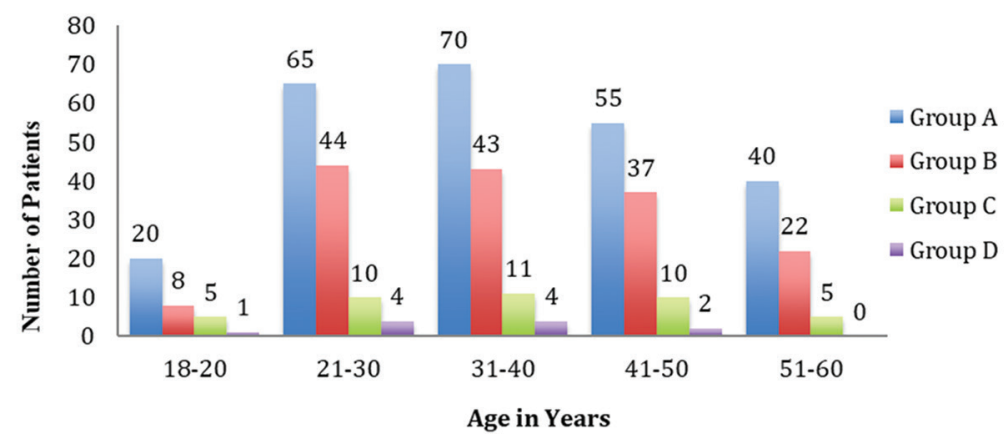

Fig. 1 Age-wise distribution of patients in the study groups.

Table 1 Demographic characteristics of patients in the study groups

\begin{tabular}{|c|c|c|c|c|c|}
\hline Variable & Group A $(n=250)$ & Group B $(n=154)$ & Group C $(n=41)$ & Group $\mathrm{D}(n=11)$ & Total $(n=456)$ \\
\hline Age $(M \pm S D), y$ & $37.5 \pm 11.6$ & $37.8 \pm 11.4$ & $36.4 \pm 12.1$ & $34.2 \pm 8.1$ & $37.4 \pm 11.5$ \\
\hline Male-to-female ratio & 156:94 & $67: 87$ & $21: 20$ & $2: 9$ & $246: 210$ \\
\hline Weight, kg & $62.5 \pm 12.8$ & $57.7 \pm 11.8$ & $51.7 \pm 9.9$ & $47.7 \pm 11.5$ & $59.6 \pm 12.8$ \\
\hline \multicolumn{6}{|l|}{ Comorbidities } \\
\hline None & $184(73.6)$ & $121(78.57)$ & $32(78.05)$ & $11(100)$ & $348(76.32)$ \\
\hline HTN & $34(13.6)$ & $10(6.49)$ & $2(4.88)$ & 0 & $46(10.09)$ \\
\hline $\mathrm{DM}$ & $8(3.2)$ & $6(3.9)$ & $2(4.88)$ & 0 & $16(3.51)$ \\
\hline Hypothyroidism & $6(2.4)$ & $1(0.65)$ & $2(4.88)$ & 0 & $9(1.97)$ \\
\hline Cardiac disease & 0 & $1(0.65)$ & 0 & 0 & $1(0.22)$ \\
\hline Psychiatric disease & $3(1.2)$ & 0 & 0 & 0 & $3(0.66)$ \\
\hline Others & $8(3.2)$ & $10(6.49)$ & $1(2.44)$ & 0 & $19(4.17)$ \\
\hline Multiple & $7(2.8)$ & $5(3.25)$ & $2(4.88)$ & 0 & $14(3.07)$ \\
\hline \multicolumn{6}{|c|}{ ASA physical status grade } \\
\hline 1 & $184(73.6)$ & $120(77.92)$ & $32(78.05)$ & $11(100)$ & $347(76.1)$ \\
\hline 2 & $64(25.6)$ & $32(20.78)$ & $9(21.95)$ & 0 & $105(23.03)$ \\
\hline 3 & $2(0.8)$ & $2(1.3)$ & 0 & 0 & $4(0.88)$ \\
\hline
\end{tabular}

Abbreviations: ASA, American Society of Anesthesiologist; DM, diabetes mellitus; HTN, hypertension; SD, standard deviation.

Note: All values are expressed as mean \pm SD or $n(\%)$.

underwent craniotomy under monitored anesthesia care for excision of tumor involving eloquent areas ( - Table 3 ). Most patients underwent simple craniotomy (58.5\%) in the supine position (67.1\%). The mean durations of surgery and anesthesia were $331.3 \pm 118.2$ minutes $(65-800)$ and $436.3 \pm$ 126.1 minutes (95-870), respectively ( - Table 5 ).

The average blood volume loss was $11 \pm 5.3 \%$ in Group A, $29.8 \% \pm 7.9 \%$ in Group B, $68.3 \pm 13.5 \%$ in Group C, and 129.1 $\pm 23.9 \%$ in Group D. The intergroup comparison showed that the blood loss between the various groups was significantly different $(p<0.001)$.

Amounts of crystalloids/colloids administered and urine output were higher through Groups A to D (-Table 4); amounts of packed red blood cells (PRBCs), fresh-frozen plasma (FFP), and platelet concentrate (PC) transfused $(p<0.001)$ also followed a similar trend. Durations of surgery and anesthesia also increased through Groups A to D ( - Table 5).

The occurrence of hemodynamic instability requiring the use of vasopressors/inotropes and other intraoperative complications such as hypothermia, acidosis, and dyselectrolytemia were significantly more among patients in the groups that had greater blood loss (-Table 6).

Almost three-fourth of the patients were extubated at the end of surgery in Group A, and the number progressively declined through Group A to Group D. A reverse trend was seen for continuation of mechanical ventilation in the postoperative period among the study groups ( $\mathbf{- T a b l e ~} \mathbf{5}$ ). In almost half of the patients who were electively ventilated at the end of surgery, the reason was either prolonged surgery or surgical concern regarding hemostasis or raised 
Table 2 Preoperative characteristics of intracranial tumors in the study groups

\begin{tabular}{|c|c|c|c|c|c|c|}
\hline Variable & $\begin{array}{l}\text { Group A } \\
(n=250), \\
n(\%)\end{array}$ & $\begin{array}{l}\text { Group B } \\
(n=154), \\
n(\%)\end{array}$ & $\begin{array}{l}\text { Group C }(n=41) \text {, } \\
n(\%)\end{array}$ & $\begin{array}{l}\text { Group D }(n=11) \text {, } \\
n(\%)\end{array}$ & $\begin{array}{l}\text { Total } \\
(n=456), \\
n(\%)\end{array}$ & $p$-Value \\
\hline \multicolumn{7}{|l|}{ Tumor location } \\
\hline Supratentorial & $116(46.4)$ & $\begin{array}{l}72 \\
(46.75)\end{array}$ & $22(53.66)$ & $4(36.36)$ & $214(46.93)$ & \multirow[t]{5}{*}{0.815} \\
\hline Posterior fossa & $28(11.2)$ & $15(9.74)$ & $3(7.32)$ & $3(27.27)$ & $49(10.75)$ & \\
\hline Cerebellopontine angle & $35(14)$ & $\begin{array}{l}21 \\
(13.64)\end{array}$ & $6(14.63)$ & $3(27.27)$ & $65(14.25)$ & \\
\hline Skull base & $56(22.4)$ & $\begin{array}{l}38 \\
(24.68)\end{array}$ & $8(19.51)$ & 1 (9.09) & 103 (22.59) & \\
\hline Intraventricular & $15(6)$ & $8(5.19)$ & $2(4.88)$ & 0 & $25(5.48)$ & \\
\hline \multicolumn{7}{|l|}{ Tumor pathology } \\
\hline Low-grade glioma & $69(27.6)$ & $\begin{array}{l}27 \\
(17.53)\end{array}$ & $4(9.76)$ & 0 & $100(21.93)$ & \multirow[t]{10}{*}{0.002} \\
\hline High-grade glioma & $19(7.6)$ & $\begin{array}{l}17 \\
(11.04)\end{array}$ & $7(17.07)$ & 1 (9.09) & $44(9.65)$ & \\
\hline Meningioma & $50(20)$ & $\begin{array}{l}54 \\
(35.06)\end{array}$ & $18(43.9)$ & $4(36.36)$ & $126(27.63)$ & \\
\hline Hemangioblastoma & $8(3.2)$ & $5(3.25)$ & $3(7.32)$ & 1 (9.09) & $17(3.73)$ & \\
\hline Schwannoma & $36(14.4)$ & $\begin{array}{l}27 \\
(17.53)\end{array}$ & $6(14.63)$ & $4(36.36)$ & $73(16.01)$ & \\
\hline Pituitary adenoma & $8(3.2)$ & $5(3.25)$ & 0 & 0 & $13(2.85)$ & \\
\hline Craniopharyngioma & $9(3.6)$ & $2(1.3)$ & 0 & 0 & $11(2.41)$ & \\
\hline Epidermoid & $22(8.8)$ & $2(1.3)$ & 0 & 0 & $24(5.26)$ & \\
\hline Medulloblastoma & $5(2)$ & $2(1.3)$ & $1(2.44)$ & 0 & $8(1.75)$ & \\
\hline Others & $24(9.6)$ & $13(8.44)$ & $2(4.88)$ & 1 (9.09) & $40(8.77)$ & \\
\hline \multicolumn{7}{|l|}{ Tumor size, $\mathrm{cm}$} \\
\hline$<5$ & $200(80)$ & $96(62.3)$ & $23(56.1)$ & $1(9.1)$ & $320(70.2)$ & $<0.001$ \\
\hline$>5$ & $50(20)$ & $58(37.7)$ & $18(43.9)$ & 10 (90.9) & $136(29.8)$ & $<0.001$ \\
\hline \multicolumn{7}{|l|}{ Other characteristics } \\
\hline Metastatic tumors & $5(2)$ & $1(0.7)$ & $1(2.4)$ & 0 & $7(1.5)$ & 0.505 \\
\hline Redo surgeries & $45(18)$ & $28(18.2)$ & $11(26.8)$ & 0 & $84(18.4)$ & 0.229 \\
\hline Mass effect & $178(71.2)$ & $\begin{array}{l}125 \\
(81.2)\end{array}$ & $37(90.2)$ & 10 (90.9) & $350(76.8)$ & 0.009 \\
\hline Midline shift & $21(8.4)$ & $23(14.9)$ & $8(19.5)$ & $2(18.2)$ & $54(11.8)$ & 0.044 \\
\hline Hydrocephalus & $44(17.6)$ & $30(19.5)$ & $11(26.8)$ & $3(27.3)$ & $88(19.3)$ & 0.427 \\
\hline Highly vascular tumors & $42(16.8)$ & $57(37)$ & $23(56.1)$ & $6(54.5)$ & $128(28.1)$ & $<0.001$ \\
\hline
\end{tabular}

Note: All values are number of patients (\%).

intracranial pressure (-Table 7). However, in Group C and Group D, the most common reason for postoperative ventilation was excessive bleeding.

During the postoperative period, fever, thrombocytopenia, coagulopathy, pulmonary infections, prolonged ICU stay (>2 days), and requirement of tracheostomy occurred with significantly higher frequency in patients of Group A through D (-Table 8). Development of dyselectrolytemia, hematoma, and requirement of surgical reexploration, blood/component transfusion, and prolonged mechanical ventilation were significantly higher in patients with greater intraoperative blood loss. In patients with postoperative hematoma, operative cavity was the most frequent location (-Table 9). The overall median duration of mechanical ventilation (-Table 10) was 17 hours (2-624 hours). The median duration of ICU stay was 1.5 days (2-864 hours), and the median hospital stay was 8 days (4-112 days). Chances of poor neurological outcome were significantly higher in patients with greater intraoperative blood loss $(p=0.01)$.

On univariate analysis, several demographics and preoperative and intraoperative variables were identified as risk factors for intraoperative bleeding. These included female gender $(p<0.001)$, presence of hypertension $(p=0.008)$, tumor size $>5 \mathrm{~cm}(p<0.001)$, tumor 
Table 3 Various intraoperative variables in the study groups

\begin{tabular}{|c|c|c|c|c|c|c|}
\hline Variable & $\begin{array}{l}\text { Group A } \\
(n=250), n(\%)\end{array}$ & $\begin{array}{l}\text { Group B } \\
(n=154), n(\%)\end{array}$ & $\begin{array}{l}\text { Group C } \\
(n=41), n(\%)\end{array}$ & $\begin{array}{l}\text { Group D } \\
(n=11), n(\%)\end{array}$ & $\begin{array}{l}\text { Total }(n=456) \text {, } \\
n(\%)\end{array}$ & $p$-Value \\
\hline \multicolumn{7}{|l|}{ Anesthetic technique } \\
\hline Inhalational & $229(91.6)$ & $148(96.1)$ & $37(90.24)$ & $11(100)$ & $425(93.2)$ & \multirow[t]{4}{*}{0.301} \\
\hline Total IV & $2(0.8)$ & 0 & 0 & 0 & $2(0.44)$ & \\
\hline IV plus inhalational & $12(4.8)$ & $6(3.9)$ & $4(9.76)$ & 0 & $22(4.82)$ & \\
\hline MAC & $7(2.8)$ & 0 & 0 & 0 & $7(1.54)$ & \\
\hline \multicolumn{7}{|l|}{ Surgical position } \\
\hline Supine & $173(69.2)$ & $102(66.23)$ & $27(65.85)$ & $4(36.36)$ & $306(67.11)$ & \multirow[t]{4}{*}{0.042} \\
\hline Lateral & $36(14.4)$ & $27(17.53)$ & $4(9.76)$ & $6(54.55)$ & $73(16.01)$ & \\
\hline Prone & $30(12)$ & $17(11.04)$ & $10(24.39)$ & $1(9.09)$ & $58(12.72)$ & \\
\hline Sitting & $11(4.4)$ & $8(5.19)$ & 0 & 0 & $19(4.17)$ & \\
\hline \multicolumn{7}{|l|}{ Surgical approach } \\
\hline Simple craniotomy & $158(63.2)$ & $79(51.3)$ & $26(63.41)$ & $4(36.36)$ & $267(58.55)$ & \multirow[t]{3}{*}{0.017} \\
\hline Extended craniotomy & $25(10)$ & $31(20.13)$ & $8(19.51)$ & $3(27.27)$ & 67 (14.69) & \\
\hline $\begin{array}{l}\text { Posterior fossa } \\
\text { craniotomy }\end{array}$ & $67(26.8)$ & $44(28.57)$ & $7(17.07)$ & $4(36.36)$ & $122(26.75)$ & \\
\hline
\end{tabular}

Abbreviations: IV, intravenous; MAC, monitored anesthesia care.

Note: All values are expressed as number of patients (\%).

Table 4 Details of blood loss and blood/component transfusion in the study groups

\begin{tabular}{|c|c|c|c|c|c|c|}
\hline Variable & $\begin{array}{l}\text { Group A } \\
(n=250)\end{array}$ & Group B $(n=154)$ & Group C $(n=41)$ & $\begin{array}{l}\text { Group D } \\
(n=11)\end{array}$ & Total $(n=456)$ & $p$-Value \\
\hline Blood loss, $\mathrm{mL}$ & $447 \pm 226$ & $1,099 \pm 360$ & $2,295 \pm 634$ & $3,854 \pm 1,179$ & $915 \pm 806$ & \\
\hline $\begin{array}{l}\text { Percentage } \\
\text { estimated blood } \\
\text { volume }\end{array}$ & $11 \pm 5.3$ & $29.8 \pm 7.9$ & $68.3 \pm 13.5$ & $129.1 \pm 23.9$ & $25.3 \pm 24.7$ & \\
\hline RBC transfusion & $32(12.8)$ & $111(72.08)$ & $38(92.68)$ & $11(100)$ & $192(42.11)$ & $<0.001$ \\
\hline RBC (units) & $\begin{array}{l}1.6 \pm 0.7(n \\
=32)\end{array}$ & $\begin{array}{l}2.1 \pm 0.9(n= \\
111)\end{array}$ & $4 \pm 1.5(n=38)$ & $\begin{array}{l}6.2 \pm 2.3(n= \\
11)\end{array}$ & $\begin{array}{l}2.6 \pm 1.6(n= \\
192)\end{array}$ & \\
\hline Cell salvage & $4(1.6)$ & 18 (11.69) & 15 (36.59) & $3(27.27)$ & $40(8.77)$ & $<0.001$ \\
\hline Autologous, mL & $\begin{array}{l}377 \pm 220(n \\
=4)\end{array}$ & $\begin{array}{l}439 \pm 291(n= \\
18)\end{array}$ & $788 \pm 492(n=15)$ & $\begin{array}{l}1,123 \pm 576(n \\
=3)\end{array}$ & $\begin{array}{l}615 \pm 443(n \\
=40)\end{array}$ & \\
\hline FFP transfusion & $3(1.2)$ & $26(16.88)$ & $26(63.41)$ & $11(100)$ & $66(14.47)$ & $<0.001$ \\
\hline FFP (units) & $\begin{array}{l}4.7 \pm 1.5(n \\
=3)\end{array}$ & $3.3 \pm 1.2(n=26)$ & $3.9 \pm 0.9(n=26)$ & $\begin{array}{l}4.5 \pm 2.3(n= \\
11)\end{array}$ & $\begin{array}{l}3.8 \pm 2.3(n= \\
66)\end{array}$ & \\
\hline $\begin{array}{l}\text { PC/PRP } \\
\text { transfusion }\end{array}$ & $3(1.2)$ & $16(10.39)$ & $20(48.78)$ & $10(90.91)$ & 49 (10.75) & $<0.001$ \\
\hline PRP (units) & $\begin{array}{l}2.3 \pm 1.5(n \\
=3)\end{array}$ & $2.7 \pm 1.1(n=16)$ & $3.3 \pm 1.1(n=20)$ & $\begin{array}{l}4.4 \pm 1.3(n= \\
10)\end{array}$ & $\begin{array}{l}3.3 \pm 1.3(n= \\
49)\end{array}$ & \\
\hline
\end{tabular}

Abbreviations: FFP, fresh frozen plasma; PC, platelet concentrate; PRP, platelet-rich plasma; RBC, red blood cell; SD, standard deviation.

Note: All values are expressed as mean \pm SD or $n(\%)$.

pathology like high-grade glioma $(p=0.004)$ and meningioma $(p<0.001)$, presence of preoperative mass effect $(p=0.002)$, preoperative midline shift $>5 \mathrm{~mm}(p=0.014)$, highly vascular tumors as seen on preoperative imaging $(p<0.001)$, extended craniotomy approach $(p=0.002)$, intraoperative use of colloids $>1,000 \mathrm{~mL}(p<0.001)$, intraoperative brain bulge $(p=0.03)$, intraoperative appearance as highly vascular tumor $(p<0.001)$, and duration of surgery $>300$ minutes $(p<0.001)(-$ Table 11).
Subsequently, multivariate logistic regression analysis was performed to find out significant risk factors for intraoperative bleeding (-Table 12). Female gender ( $p=0.002$ ), presence of hypertension $(p=0.017)$, tumor size $>5 \mathrm{~cm}(p=0.008)$, preoperative and intraoperative appearance of highly vascular tumors ( $p=0.041$ and $p=0.003$, respectively), extended craniotomy approach $(p=0.009)$, intraoperative use of colloids $>1,000 \mathrm{~mL}(p<0.001)$, and duration of surgery $>300$ minutes $(p<0.001)$ were found to 
Table 5 Various intraoperative parameters in the study group

\begin{tabular}{|c|c|c|c|c|c|c|}
\hline Variable & $\begin{array}{l}\text { Group A } \\
(n=250)\end{array}$ & $\begin{array}{l}\text { Group B } \\
(n=154)\end{array}$ & $\begin{array}{l}\text { Group C } \\
(n=41)\end{array}$ & Group D $(n=11)$ & Total $(n=456)$ & $p$-Value \\
\hline $\begin{array}{l}\text { Duration of sur- } \\
\text { gery, min }\end{array}$ & $282.6 \pm 91.6$ & $374.9 \pm 110.7$ & $429.7 \pm 133.8$ & $461.8 \pm 144.1$ & $331.3 \pm 118.2$ & \\
\hline $\begin{array}{l}\text { Duration of anes- } \\
\text { thesia, min }\end{array}$ & $384.7 \pm 103.1$ & $483.7 \pm 112.8$ & $532.9 \pm 137.2$ & $583.2 \pm 162$ & $436.3 \pm 126.1$ & \\
\hline Crystalloids, mL & $\begin{array}{l}3,498.4 \pm \\
969.9\end{array}$ & $\begin{array}{l}4,310.1 \pm \\
1,163.9\end{array}$ & $5,195.1 \pm 1,122.9$ & $7,090.9 \pm 3,088.8$ & $\begin{array}{l}4,011.7 \pm \\
1,349.5\end{array}$ & \\
\hline Colloids, mL & $\begin{array}{l}647.4 \pm 255.8 \\
(n=171)\end{array}$ & $\begin{array}{l}856.9 \pm 359.5 \\
(n=151)\end{array}$ & $\begin{array}{l}1,219.5 \pm 388.1 \\
(n=41)\end{array}$ & $\begin{array}{l}1,400 \pm 614.6 \\
(n=10)\end{array}$ & $\begin{array}{l}815.3 \pm 385 \\
(n=373)\end{array}$ & \\
\hline Urine output, mL & $\begin{array}{l}1,571.2 \pm \\
798.1\end{array}$ & $\begin{array}{l}1,844.9 \pm \\
901.5\end{array}$ & $2,270.7 \pm 1,205.1$ & $3,136.4 \pm 1,434.9$ & $1,764.3 \pm 941.3$ & \\
\hline Reversal & $191(76.4)$ & $62(40.26)$ & $5(12.2)$ & 0 & $258(56.58)$ & $<0.001$ \\
\hline Extubation & $184(73.6)$ & $57(37.01)$ & $5(12.2)$ & 0 & 246 (53.95) & $<0.001$ \\
\hline Ventilation & $67(26.8)$ & 97 (62.99) & $36(87.8)$ & $11(100)$ & $211(46.27)$ & $<0.001$ \\
\hline
\end{tabular}

Note: All values are expressed as mean \pm SD or $n(\%)$.

Table 6 Intraoperative complications

\begin{tabular}{|c|c|c|c|c|c|c|}
\hline Variable & $\begin{array}{l}\text { Group A } \\
(n=250), n(\%)\end{array}$ & $\begin{array}{l}\text { Group B } \\
(n=154), n(\%)\end{array}$ & $\begin{array}{l}\text { Group C } \\
(n=41), n(\%)\end{array}$ & $\begin{array}{l}\text { Group D } \\
(n=11), n(\%)\end{array}$ & $\begin{array}{l}\text { Total }(n=456), \\
n(\%)\end{array}$ & $p$-Value \\
\hline $\begin{array}{l}\text { Hemodynamic } \\
\text { instability }\end{array}$ & $1(0.4)$ & $2(1.3)$ & $8(19.5)$ & $6(54.5)$ & $17(3.7)$ & $<0.001$ \\
\hline $\begin{array}{l}\text { Inotrope/vasopressor } \\
\text { use }\end{array}$ & $1(0.4)$ & $1(0.7)$ & 0 & $6(54.5)$ & $8(1.7)$ & $<0.001$ \\
\hline Hypothermia & 0 & 0 & $12(29.3)$ & $9(81.8)$ & $21(4.6)$ & $<0.001$ \\
\hline Dyselectrolytemia & 0 & 0 & 0 & $1(9.1)$ & $1(0.2)$ & 0.024 \\
\hline Acidosis & 0 & 0 & $2(4.9)$ & 0 & $2(0.4)$ & 0.013 \\
\hline Difficult hemostasis & $1(0.4)$ & 0 & $1(2.4)$ & 0 & $2(0.4)$ & 0.215 \\
\hline
\end{tabular}

Note: All values are number of patients (\%).

Table 7 Various reasons for elective ventilation in the study patients

\begin{tabular}{|c|c|c|c|c|c|c|}
\hline $\begin{array}{l}\text { Reason for } \\
\text { ventilation }\end{array}$ & $\begin{array}{l}\text { Group A } \\
(n=250), n(\%)\end{array}$ & $\begin{array}{l}\text { Group B } \\
(n=154), n(\%)\end{array}$ & $\begin{array}{l}\text { Group C } \\
(n=41), n(\%)\end{array}$ & $\begin{array}{l}\text { Group D } \\
(n=11), n(\%)\end{array}$ & $\begin{array}{l}\text { Total }(n=456), \\
n(\%)\end{array}$ & $p$-Value \\
\hline $\begin{array}{l}\text { Preoperative LCN } \\
\text { palsy }\end{array}$ & $5(2)$ & $11(7.14)$ & $1(2.44)$ & 0 & $17(3.73)$ & \multirow[t]{8}{*}{$<0.001$} \\
\hline Brainstem handling & $16(6.4)$ & $6(3.9)$ & 0 & $2(18.18)$ & $24(5.26)$ & \\
\hline Bleeding & 0 & $2(1.3)$ & $19(46.34)$ & $9(81.82)$ & $30(6.58)$ & \\
\hline $\begin{array}{l}\text { Intraoperative brain } \\
\text { bulge }\end{array}$ & $3(1.2)$ & $2(1.3)$ & 0 & 0 & $5(1.1)$ & \\
\hline Residual tumor & $3(1.2)$ & $13(8.44)$ & 0 & 0 & $16(3.51)$ & \\
\hline Prolonged surgery & $9(3.6)$ & $29(18.83)$ & $11(26.83)$ & 0 & $49(10.75)$ & \\
\hline $\begin{array}{l}\text { Not obeying } \\
\text { commands }\end{array}$ & $8(3.2)$ & $5(3.25)$ & 0 & 0 & $13(2.85)$ & \\
\hline Surgical factors & $23(9.2)$ & $29(18.83)$ & $5(12.2)$ & 0 & $57(12.5)$ & \\
\hline
\end{tabular}

Abbreviation: LCN, lower cranial nerve.

Note: All values are number of patients (\%).

be the most predictive variables for intraoperative blood loss (-Table 11). However, tumor pathology such as meningioma (OR: 2.34; CI: $1.5-3.5 ; p=0.065$ ) and tumor location at skull base (OR: $1.02 ; \mathrm{CI}: 0.6-1.6 ; p=0.063$ ) also demonstrated weak significance (-Table 12 ).
The majority of patients had good neurological recovery (71.3\%). Around 19\% patients had moderate disability and $5.9 \%$ had severe disability. Only two patients $(0.4 \%)$ were discharged in persistent vegetative state. The mortality rate in our study population was $2.8 \%$ (-Fig. 2 ). 
Table 8 Postoperative complications in the study patients

\begin{tabular}{|c|c|c|c|c|c|c|}
\hline Variable & $\begin{array}{l}\text { Group A } \\
(n=250), \\
n(\%)\end{array}$ & $\begin{array}{l}\text { Group B } \\
(n=154), \\
n(\%)\end{array}$ & $\begin{array}{l}\text { Group C } \\
(n=41), n(\%)\end{array}$ & $\begin{array}{l}\text { Group D } \\
(n=11), n(\%)\end{array}$ & $\begin{array}{l}\text { Total } \\
(n=456), \\
n(\%)\end{array}$ & $p$-Value \\
\hline Anemia ( $\mathrm{Hb}<8 \mathrm{~g} \%)$ & $5(2)$ & $5(3.3)$ & $4(9.8)$ & 0 & $14(3.1)$ & 0.091 \\
\hline $\begin{array}{l}\text { Thrombocytopenia (platelet } \\
\text { count }<100,000 / \mathrm{mm}^{3} \text { ) }\end{array}$ & $5(2)$ & $15(9.7)$ & $13(31.7)$ & $6(54.5)$ & $39(8.5)$ & $<0.001$ \\
\hline Leukocytosis (TLC >11,000/mm³) & 149 (59.6) & $98(63.6)$ & $23(56.1)$ & $7(63.6)$ & $277(60.7)$ & 0.781 \\
\hline Fever & $58(23.2)$ & $66(42.9)$ & $21(51.2)$ & $10(90.9)$ & 155 (33.9) & $<0.001$ \\
\hline Dyselectrolytemia & $4(1.6)$ & $10(6.5)$ & $5(12.2)$ & $1(9.1)$ & $20(4.4)$ & 0.003 \\
\hline Deranged renal function tests & $1(0.4)$ & $1(0.6)$ & 0 & 0 & $2(0.4)$ & 1.000 \\
\hline Deranged liver function tests & 0 & 0 & $1(2.4)$ & 0 & $1(0.2)$ & 0.114 \\
\hline Coagulopathy & $2(0.8)$ & $3(1.9)$ & $6(14.6)$ & $2(18.2)$ & $13(2.8)$ & $<0.001$ \\
\hline Hemodynamic instability & $3(1.2)$ & $1(0.7)$ & $1(2.4)$ & 0 & $5(1.1)$ & 0.544 \\
\hline Inotrope/vasopressor use & $3(1.2)$ & $1(0.7)$ & $1(2.4)$ & 0 & $5(1.1)$ & 0.544 \\
\hline Transfusions & $37(14.8)$ & $56(36.4)$ & $30(73.2)$ & $7(63.6)$ & $130(28.5)$ & $<0.001$ \\
\hline Multiorgan dysfunction & $2(0.8)$ & $4(2.6)$ & $1(2.4)$ & 0 & $7(1.5)$ & 0.378 \\
\hline Pulmonary infection & $2(0.8)$ & $9(5.8)$ & $2(4.9)$ & $1(9.1)$ & $14(3.1)$ & 0.006 \\
\hline Sepsis & 0 & $3(1.95)$ & 0 & 0 & $3(0.66)$ & 0.161 \\
\hline Urinary tract infection & $1(0.4)$ & $1(0.65)$ & 0 & 0 & $2(0.44)$ & 1.000 \\
\hline Bloodstream infection & 0 & $1(0.65)$ & $1(2.44)$ & 0 & $2(0.44)$ & 0.116 \\
\hline Wound infection & 0 & $2(1.3)$ & 0 & 0 & $2(0.44)$ & 0.329 \\
\hline Reexploration for hematoma & $6(2.4)$ & $4(2.6)$ & $5(12.2)$ & $1(9.09)$ & $16(3.51)$ & 0.018 \\
\hline Hematoma & $29(11.6)$ & $28(18.18)$ & $12(29.27)$ & $3(27.27)$ & $72(15.79)$ & 0.011 \\
\hline Tracheostomy & $5(2)$ & $14(9.09)$ & $4(9.76)$ & $3(27.27)$ & $26(5.7)$ & $<0.001$ \\
\hline Prolonged ventilation & $13(5.2)$ & $16(10.4)$ & $9(21.9)$ & $2(18.2)$ & $40(8.7)$ & $<0.001$ \\
\hline Prolonged ICU stay & $13(5.2)$ & $23(14.9)$ & $8(19.5)$ & $4(36.4)$ & $48(10.5)$ & $<0.001$ \\
\hline Prolonged hospital stay & $112(44.8)$ & $95(61.7)$ & $32(78)$ & $9(81.8)$ & $248(54.4)$ & 0.054 \\
\hline Poor neurological outcome & $15(6)$ & $17(11)$ & $8(19.5)$ & $2(18.2)$ & $42(9.2)$ & 0.013 \\
\hline
\end{tabular}

Abbreviations: Hb, hemoglobin; ICU, intensive care unit; TLC, total leukocyte count.

Note: All values are number of patients (\%).

Table 9 Types of postoperative hematoma in the study patients

\begin{tabular}{|c|c|c|c|c|c|c|}
\hline Type of hematoma & $\begin{array}{l}\text { Group A } \\
(n=250), n(\%)\end{array}$ & $\begin{array}{l}\text { Group B } \\
(n=154), n(\%)\end{array}$ & $\begin{array}{l}\text { Group C } \\
(n=41), n(\%)\end{array}$ & $\begin{array}{l}\text { Group D }(n=11) \text {, } \\
n(\%)\end{array}$ & $\begin{array}{l}\text { Total }(n=456) \text {, } \\
n(\%)\end{array}$ & $p$-Value \\
\hline Extradural & $6(2.4)$ & $4(2.6)$ & $2(4.88)$ & $1(9.09)$ & $13(2.85)$ & \multirow[t]{7}{*}{0.106} \\
\hline Subdural & 0 & 0 & $1(2.44)$ & 0 & $1(0.22)$ & \\
\hline Intracerebral & $1(0.4)$ & $2(1.3)$ & $1(2.44)$ & 1 (9.09) & $5(1.1)$ & \\
\hline Subarachnoid & $1(0.4)$ & 0 & 0 & 0 & $1(0.22)$ & \\
\hline Intraventricular & $4(1.6)$ & $6(3.9)$ & $2(4.88)$ & 0 & $12(2.63)$ & \\
\hline Tumor cavity & $17(6.8)$ & $15(9.74)$ & $5(12.2)$ & 1 (9.09) & $38(8.33)$ & \\
\hline Multiple & $2(0.8)$ & $1(0.65)$ & 0 & 0 & $3(0.66)$ & \\
\hline
\end{tabular}

Note: All values are number of patients (\%).

\section{Discussion}

In this retrospective study, we found that the patients who had $>20 \%$ estimated blood volume loss had greater incidence of complications, and the rate was higher with increasing blood loss. Blood transfusion during neurosurgery was found to strongly correlate with postoperative complications in a large retrospective study by Rolston et al. ${ }^{15}$
Only $42.1 \%$ of patients required blood or blood product transfusion in our study, which was slightly less compared with the audit by Bhatnagar et $\mathrm{al}^{16}{ }^{16}$ where they found that $48 \%$ of their patients with intracranial tumors required transfusion. In our study, 32 (12.8\%) patients who had $<20 \%$ estimated blood volume loss also received blood transfusion; the reason of transfusion was not mentioned in all anesthesia 
Table 10 Postoperative parameters in the study groups

\begin{tabular}{|l|l|l|l|l|l|}
\hline Variable & Group A $(n=250)$ & Group B $(n=154)$ & Group C $(n=41)$ & Group D $(n=11)$ & Total $(n=456)$ \\
\hline Duration of MV, d & $1.4 \pm 2.4(n=67)$ & $2.3 \pm 4.7(n=97)$ & $3 \pm 5.4(n=36)$ & $1.4 \pm 1.7(n=11)$ & $2.1 \pm 4.2(n=211)$ \\
\hline ICU stay, d & $2 \pm 2.7$ & $3.7 \pm 5.3$ & $4.2 \pm 5.1$ & $5.1 \pm 3.5$ & $2.9 \pm 4.1$ \\
\hline Hospital stay, d & $9.4 \pm 7$ & $13.6 \pm 15.2$ & $14.9 \pm 13.4$ & $15.3 \pm 7.1$ & $11.5 \pm 11.2$ \\
\hline
\end{tabular}

Abbreviations: ICU, intensive care unit; MV, mechanical ventilation.

Note: All values are expressed as mean \pm SD.

Table 11 Predictors of $>20 \%$ blood volume loss on univariate analysis

\begin{tabular}{|l|l|l|l|}
\hline Variable & OR & $95 \%$ Cl & $p$-Value \\
\hline Female gender & 2.14 & $1.468-3.115$ & 0.0001 \\
\hline HTN & 0.39 & $0.198-0.78$ & 0.008 \\
\hline Tumor size $>5 \mathrm{~cm}$ & 2.87 & $1.892-4.344$ & $<0.001$ \\
\hline HGG & 2.93 & $1.409-6.088$ & 0.004 \\
\hline Meningioma & 3.38 & $1.944-5.888$ & $<0.001$ \\
\hline Preoperative mass effect & 2.05 & $1.293-3.237$ & 0.0018 \\
\hline Preoperative midline shift & 2.08 & $1.162-3.721$ & 0.0123 \\
\hline Vascular tumor (imaging) & 3.8 & $1.794-8.054$ & $<0.001$ \\
\hline Extended craniotomy approach & 2.44 & $1.402-4.229$ & 0.002 \\
\hline Colloids $>1,000$ mL & 23.33 & $8.371-65.019$ & $<0.001$ \\
\hline Intraoperative brain bulge & 1.75 & $1.055-2.918$ & 0.03 \\
\hline High vascularity (appearance) & 13.34 & $6.282-28.315$ & $<0.001$ \\
\hline Surgery duration $>$ 300 min & 1.01 & $1.007-1.012$ & $<0.001$ \\
\hline Abbrions: Cl, confide & \\
\hline
\end{tabular}

Abbreviations: $\mathrm{Cl}$, confidence interval; HTN, hypertension; HGG, high-grade glioma; OR, odds ratio.

Table 12 Most predictive variables of intraoperative blood loss on multivariate regression analysis

\begin{tabular}{|l|l|l|l|}
\hline Variable & OR & $95 \% \mathrm{Cl}$ & $p$-Value \\
\hline Female gender & 2.44 & $1.37-4.35$ & 0.002 \\
\hline HTN & 0.33 & $0.13-0.82$ & 0.017 \\
\hline Tumor size $>5 \mathrm{~cm}$ & 2.3 & $1.24-4.28$ & 0.008 \\
\hline Vascular tumor (imaging) & 2.31 & $1.32-4.05$ & 0.003 \\
\hline Extended craniotomy approach & 0.63 & $0.44-0.89$ & 0.009 \\
\hline Colloids $>1,000$ mL & 27.32 & $6.68-111.68$ & $<0.001$ \\
\hline High vascularity (appearance) & 2.09 & $1.29-3.37$ & 0.003 \\
\hline Surgery duration >300 min & 1.01 & $1-1.01$ & $<0.001$ \\
\hline Pathology-meningioma & 0.51 & $0.24-1.04$ & 0.065 \\
\hline Location-skull base & 0.53 & $0.26-1.04$ & 0.065 \\
\hline
\end{tabular}

Abbreviations: $\mathrm{Cl}$, confidence interval; HTN, hypertension; OR, odds ratio.

records but might be due to low baseline hemoglobin, presence of cardiorespiratory comorbidity, rapidity of blood loss, or alarming clinical signs.

Intraoperative complications such as hemodynamic instability requiring inotrope/vasopressor support, hypothermia, and need for elective ventilation postsurgery were more frequent in patients who sustained $>50 \%$ estimated blood volume loss. Patients who had $>20 \%$ estimated blood volume loss required infusion of more crystalloids and colloids intraoperatively. Colloid infusion more than $1 \mathrm{~L}$ was found more commonly in Groups C and D. Similarly, the requirement for blood components such as FFP and PC were also higher in these patients. Piastra et al found that infants who had blood loss exceeding their estimated total blood volume during excision of brain tumor required significantly more intraoperative cardiovascular support. ${ }^{13}$ In addition, they also had significantly higher ICU length of stay and postoperative mechanical ventilation requirement.

In our study, anemia in the postoperative period was slightly more in patients who had $>20 \%$ estimated blood volume loss (4.4 vs. $2 \%$ ). However, this difference was not statistically significant. This probably shows adequacy of transfusion 


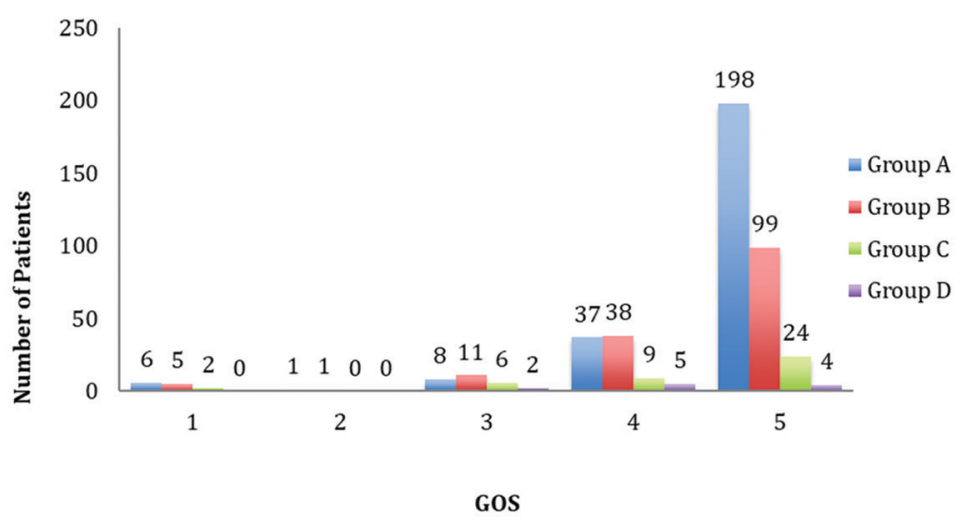

Fig. 2 Glasgow Outcome Scale (GOS) ( 1 = death, 2 = persistent vegetative state, 3 = severe disability, $4=$ moderate disability, $5=$ good recovery).

efforts during the intraoperative period. Thrombocytopenia was more common in patients who had $>20 \%$ estimated blood volume loss, almost varying linearly with the amount of blood lost. This may be due to the dilutional effect due to infusion of IV fluids, PRBCs, and FFP (poor in platelets).

Postoperatively, fever was more common in patients who had more blood loss, which could be either reactionary to blood transfusion or an early indicator of onset of infection, both of which were more common in patients who had more severe intraoperative bleeding.

Postoperative infections were also positively associated with blood loss and transfusion with most of the infections (pulmonary, bloodstream, wound/surgical site, sepsis, etc.) more frequent in patients who had major blood loss and blood transfusion. Huber-Wagner et $\mathrm{al}^{17}$ and Working Group on Polytrauma of the German Trauma Society prospectively studied massive blood transfusion and outcome in 1,062 polytrauma patients and found that $\sim 15 \%$ of polytrauma patients who received massive blood transfusion showed risk of organ failure, sepsis, and death correlating with increasing transfusion amount. In our study, risk of postoperative pulmonary infection strongly correlated with intraoperative blood loss and blood/component transfusion (OR: 24; CI: 1.76-327.3; $p=0.017$ ).

The increased incidence of perioperative complications in patients who had $>20 \%$ estimated blood volume loss resulted in increased morbidity reflected by prolonged duration of mechanical ventilation, ICU stay, and hospital stay in these patients. Bochicchio et $\mathrm{al}^{18}$ found that in trauma patients admitted to ICU, blood product transfusion was associated with a significantly greater infection rate, hospital and ICU stay, ventilator days, and mortality with a dose-dependent correlation.

On regression analysis, several factors were found to positively correlate as risks for intraoperative bleeding. Although all other factors are convincing for their presence, observation of female gender as a significant predictor of intraoperative blood loss is surprising. We did not find any study with similar finding in the literature; therefore, it needs to be further confirmed in larger studies.
Massive intraoperative blood loss has been found to increase the risk of perioperative death by up to eightfold in some studies. ${ }^{1}$ In our study, we found certain predictors of blood loss, of which female gender, tumor size $>5 \mathrm{~cm}$, high vascularity of tumor assessed on the basis of preoperative imaging as well as intraoperative appearance, colloid transfusion $>1 \mathrm{~L}$, and surgery duration $>300$ minutes were most notable.

There are certain limitations of this study, including those inherent to all retrospective study designs. Second, all our patients were adults. Third, we included only patients undergoing elective neurosurgery.

\section{Conclusions}

Intracranial tumor excision can be associated with major blood loss and require transfusion of large volumes of blood and blood products. This study has identified various factors for intraoperative blood loss during brain tumor surgery. By knowing these predictors, the anesthesiologist can anticipate major blood loss and be prepared to avoid intraoperative hemodynamic instabilities, hypothermia, and acidosis, and make judicious use of blood and blood products, which may go a long way in improving patient outcome.

\section{Funding \\ None. \\ Conflict of Interest \\ None declared.}

\section{References}

1 Karkouti K, Wijeysundera DN, Yau TM, et al. The independent association of massive blood loss with mortality in cardiac surgery. Transfusion 2004;44(10):1453-1462

2 Blumberg N. Deleterious clinical effects of transfusion immunomodulation: proven beyond a reasonable doubt. Transfusion 2005;45(2)(suppl):33S-39S, discussion 39S-40S

3 Blajchman MA, Vamvakas EC. The continuing risk of transfusion-transmitted infections. $N$ Engl J Med 2006;355(13):1303-1305 
4 Triulzi DJ, Vanek K, Ryan DH, Blumberg N. A clinical and immunologic study of blood transfusion and postoperative bacterial infection in spinal surgery. Transfusion 1992;32(6):517-524

5 Hill GE, Frawley WH, Griffith KE, Forestner JE, Minei JP. Allogeneic blood transfusion increases the risk of postoperative bacterial infection: a meta-analysis. J Trauma 2003;54(5):908-914

6 Palomo Sanchez JC, Jimenez C, Moreno Gonzalez E, et al. Effects of intraoperative blood transfusion on postoperative complications and survival after orthotopic liver transplantation. Hepatogastroenterology 1998;45(22):1026-1033

7 Manning-Geist BL, Alimena S, Del Carmen MG, et al. Infection, thrombosis, and oncologic outcome after interval debulking surgery: does perioperative blood transfusion matter? Gynecol Oncol 2019;153(1):63-67

8 Gurusamy KS, Pissanou T, Pikhart H, Vaughan J, Burroughs AK, Davidson BR. Methods to decrease blood loss and transfusion requirements for liver transplantation. Cochrane Database Syst Rev 2011;( 12):CD009052

9 Lynn M, Jeroukhimov I, Klein Y, Martinowitz U. Updates in the management of severe coagulopathy in trauma patients. Intensive Care Med 2002;28(suppl 2):S241-S247

10 Helling TS, Blondeau B, Wittek BJ. Perioperative factors and outcome associated with massive blood loss during major liver resections. HPB (Oxford) 2004;6(3):181-185

11 Isbister JP, Shander A, Spahn DR, Erhard J, Farmer SL, Hofmann A. Adverse blood transfusion outcomes: establishing causation. Transfus Med Rev 2011;25(2):89-101
12 Spahn DR, Shander A, Hofmann A, Berman MF. More on transfusion and adverse outcome: it's time to change. Anesthesiology 2011;114(2):234-236

13 Piastra M, Di Rocco C, Caresta E, et al. Blood loss and shortterm outcome of infants undergoing brain tumour removal. J Neurooncol 2008;90(2):191-200

14 Warner LL, Dowdy SC, Martin JR, et al. The impact of perioperative packed red blood cell transfusion on survival in epithelial ovarian cancer. Int J Gynecol Cancer 2013;23(9):1612-1619

15 Rolston JD, Han SJ, Lau CY, Berger MS, Parsa AT. Frequency and predictors of complications in neurological surgery: national trends from 2006 to 2011. J Neurosurg 2014;120(3):736-745

16 Bhatnagar S, Udaya IB, Rao GS. An audit of blood transfusion in elective neurosurgery. Indian J Anaesth 2007;51:200-204

17 Huber-Wagner S, Qvick M, Mussack T, et al. Working Group on Polytrauma of German Trauma Society (DGU). Massive blood transfusion and outcome in 1062 polytrauma patients: a prospective study based on the Trauma Registry of the German Trauma Society. Vox Sang 2007;92(1):69-78

18 Bochicchio GV, Napolitano L, Joshi M, Bochicchio K, Meyer W, Scalea TM. Outcome analysis of blood product transfusion in trauma patients: a prospective, risk-adjusted study. World J Surg 2008;32(10):2185-2189 